\title{
Adaptive Transfer Learning of Cross-Spatiotemporal Canonical Correlation Analysis for Plant-Wide Process Monitoring
}

Hongchao Cheng 1,2 , Yiqi Liu1* , Daoping Huang1, Yongping Pan 3, Qilin Wang2

1 School of Automation Science and Engineering, South China University of Technology, Guangzhou 510640, China. Hongchao.Cheng@uts.edu.au

2 Centre for Technology in Water and Wastewater, School of Civil and Environmental Engineering, University of Technology Sydney, Ultimo, NSW 2007, Australia.

3 Department of Biomedical Engineering, National University of Singapore, Singapore Medical Drive, 117575, Singapore.

Corresponding author: E-mail addresses: aulyq@scut.edu.cn Tel: +86 20 87114189; Fax: +86 20 87114189 


\section{Supplementary Material A}

Table S1. Detection results for random variation fault

\begin{tabular}{|c|c|c|c|c|c|c|c|}
\hline PEI & & PCA-T $^{2}$ & ${\mathrm{CCA}-\mathrm{T}^{2}}^{2}$ & SVM & SVMt & TrSVM & Proposed \\
\hline \multirow{3}{*}{ FAR } & $2 \%$ & 0.2241 & 0.0603 & 0.5833 & 0.2759 & 0.2874 & 0.0029 \\
\hline & $6 \%$ & 0.3179 & 0.0309 & 0.4105 & 0.2932 & 0.3086 & $\mathbf{0}$ \\
\hline & $10 \%$ & 0.2724 & 0.0897 & 0.3787 & 0.3289 & 0.3355 & 0.0133 \\
\hline \multirow{3}{*}{ MAR } & $2 \%$ & 0.0928 & 0.366 & $\mathbf{0}$ & $\mathbf{0}$ & $\mathbf{0}$ & 0.1675 \\
\hline & $6 \%$ & 0.0165 & 0.3077 & $\mathbf{0}$ & $\mathbf{0}$ & $\mathbf{0}$ & 0.033 \\
\hline & $10 \%$ & $\mathbf{0}$ & 0.3842 & 0 & 0 & $\mathbf{0}$ & 0.0147 \\
\hline \multirow{3}{*}{ PAR } & $2 \%$ & 0.1453 & 0.2437 & 0.2333 & 0.1103 & 0.1149 & 0.1017 \\
\hline & $6 \%$ & 0.1371 & 0.197 & 0.1642 & 0.1173 & 0.1235 & 0.0198 \\
\hline & $10 \%$ & 0.109 & 0.2664 & 0.1515 & 0.1316 & 0.1342 & 0.0141 \\
\hline \multirow{3}{*}{ Acc } & $2 \%$ & 0.8451 & 0.7785 & 0.7242 & 0.8696 & 0.8641 & 0.9103 \\
\hline & $6 \%$ & 0.8416 & 0.8227 & 0.8067 & 0.8619 & 0.8547 & 0.9826 \\
\hline & $10 \%$ & 0.8723 & 0.7539 & 0.8224 & 0.8458 & 0.8427 & 0.986 \\
\hline \multicolumn{8}{|c|}{ Table S2. Detection results for sticking fault } \\
\hline \multirow[t]{2}{*}{ PEI } & & $\mathrm{PCA}-\mathrm{T}^{2}$ & ${\mathrm{CCA}-\mathrm{T}^{2}}^{2}$ & SVM & SVMt & TrSVM & Proposed \\
\hline & $2 \%$ & 0.2241 & 0.4052 & 0.908 & 0.0029 & 0.0144 & $\overline{0}$ \\
\hline \multirow[t]{2}{*}{ FAR } & $6 \%$ & 0.142 & 0.4815 & 0.6235 & 0.0525 & 0.1173 & $\mathbf{0}$ \\
\hline & $10 \%$ & 0.2292 & 0.5581 & 0.5349 & $\mathbf{0}$ & 0.0299 & 0.0698 \\
\hline \multirow{3}{*}{ MAR } & $2 \%$ & 0.5464 & 0.6057 & 0.0052 & 0.933 & 0.5799 & 0.1933 \\
\hline & $6 \%$ & 0.5082 & 0.4753 & 0.0082 & 0.2418 & 0.1319 & 0.1401 \\
\hline & $10 \%$ & 0.5103 & 0.3548 & 0.0352 & 0.9971 & 0.3519 & 0.0264 \\
\hline \multirow{3}{*}{ PAR } & $2 \%$ & 0.4175 & 0.5255 & 0.3663 & 0.5609 & 0.3537 & 0.116 \\
\hline & $6 \%$ & 0.3617 & 0.4778 & 0.2543 & 0.166 & 0.126 & 0.0841 \\
\hline & $10 \%$ & 0.3979 & 0.4362 & 0.2351 & 0.5982 & 0.2231 & 0.0437 \\
\hline \multirow{3}{*}{ Acc } & $2 \%$ & 0.606 & 0.4891 & 0.5679 & 0.5068 & 0.6875 & 0.8981 \\
\hline & $6 \%$ & 0.6642 & 0.5218 & 0.702 & 0.8474 & 0.875 & 0.9259 \\
\hline & $10 \%$ & 0.6215 & 0.5498 & 0.7305 & 0.4704 & 0.7991 & $\mathbf{0 . 9 5 3 3}$ \\
\hline
\end{tabular}

Table S1 and Table S2 represent the detection results of fault 2 and fault 3, respectively. When PR value is $2 \%$, the accuracy of eight methods for fault 2 can be sorted by the following sequence: CS-AdBoostTrLM $>$ SVMt $>$ TrSVM $>$ PCA-T2 > CCA-T2 > SVM. We can find that the performance of TrSVM is not always better than SVMt, which indicates that the domain mismatch may causes information cannot be transferred effectively. However, TrSVM has better performance than the SVM 
method. When PR value is $10 \%$, the accuracy of CS-AdBoostTrLM for fault 3 is 95.33\%. The accuracy of TrSVM and SVMt are $79.91 \%$ and $47.04 \%$, respectively, which are lower than the CS-AdBoostTrLM method. 